\title{
Author's response to: Bozzetti F, nutrition, hydration, and patient's preferences at the end of life
}

\author{
Johannes Bükki ${ }^{1}$
}

Received: 16 February 2015 / Accepted: 9 March 2015 / Published online: 20 March 2015

(C) Springer-Verlag Berlin Heidelberg 2015

\section{Dear Editor,}

We appreciate the comments made by Dr. Bozzetti. Moreover, the rather restrictive current recommendations on artificial nutrition (AN) in patients with advanced cancer- " parenteral nutrition is ineffective and probably harmful in non-aphagic oncological patients in whom there is no gastrointestinal reason for intestinal failure (grade A)" [1] —are helpful in decision making and end-of-life (EoL) discussions.

We also fully agree that prognostication and definitions of EoL are relevant for both study design and decision making. However, the focus of this work was on the preferences of symptomatic cancer patients regarding their own EoL care rather than on illness trajectories. Therefore, in the opening statement of the questionnaire, the EoL situation was defined by a common physical phenomenon ("no longer able to eat or drink by themselves") rather than by a time frame.

Regarding the point on artificial hydration (AH) in the last days of life, AH may precipitate edema, effusions, tracheal secretions, and dyspnea, while beneficial effects on dehydration symptoms are inconsistent [2]. Thus, we routinely establish the individual risk-benefit ratio, frequently recommend against $\mathrm{AH}$, suggest alternatives (such as mouth care), and aim at reaching a broad consensus of all parties involved.

Conflict of interest The author declares no conflict of interest.

\section{References}

1. Bozzetti F, Arends J, Lundholm K, Micklewright A, Zurcher G, Muscaritoli M, ESPEN (2009) ESPEN guidelines on parenteral nutrition: non-surgical oncology. Clin Nutr 28:445-454

2. Nakajima N, Satake N, Nakaho T (2014) Indications and practice of artificial hydration for terminally ill cancer patients. Curr Opin Supp Palliat Care 8:358-363
Johannes Bükki

dr.j.buekki@hospiz-da-sein.de

1

Hospice Care DaSein, Karlstr. 56-58, 80333 Munich, Germany 